\title{
Analysis on the Characteristics, Construction and Application of Intelligent Library
}

\author{
He Ying \\ Dept. Name of Organization: Hunan Railway Professional Technology College \\ Zhuzhou, Hunan, China, 412001 \\ emaill:8774080@qq.com
}

Keywords: Wisdom library; Property Internet; Construction

\begin{abstract}
Nowadays, the research of intelligent library has changed from technical to the core idea of human nature. Intelligent library refers the application of intelligent sensing environment and integrated information service platform. Its characteristics are technology-based, service-cored, environment-perception- overall, network seamless, data-massive and learning environment-open. The core concept is people-oriented and focusing on users' need. There are three levels. They are the perception layer, network layer and application layer. Intelligent library is the future developing mode instead of present library.

In the macro philosophy wisdom earth, the library's existence and operation forms will be revolutionary change. Part of the business of the library will undergo major changes, also will produce new content; management of the library will be the formation of integration management system advantages, and to make related decisions more scientific basis; resources construction in library, information literacy, information research advisory work with the development of the Internet technology and the continuous adjustment library; service and management will show the wisdom, transparent, seamless and personalized features, to change the service system and the library oriented to intelligence service platform of user oriented library; the library will be through natural, efficient human-computer interaction, seamless mobile information service and transparent, and by for perception of the upper and lower, provide a more personalized intelligent service for users. So, the objective requirements of library network environment to produce wisdom library.
\end{abstract}

\section{The origin and development of wisdom library}

In 2008, America IBM president and CEO Pang Mingsheng entitled "wisdom of the earth: the next generation of leadership agenda" speech was first proposed in the "wisdom of the earth" concept. In 2009, Obama became USA president to give a positive response to this idea. The concept of "wisdom of the earth" was put forward, is highly concerned American from all walks of life, even the analysis thinks this national strategy IBM's vision is very likely to rise to USA, and caused a sensation in the world.

In 1995, Bill Gates first proposed the prototype object is in the book "the road ahead". In 1999, the concept of things was formally put forward and quickly spread to the world. With the intelligent technology for complex associated information based wisdom city (Smart city) is a new concept and a new practice especially future development since the beginning of twenty-first Century in the global expansion of the city at the end of the twentieth Century.

Library science understanding of wisdom library unceasingly thorough, the present stage began to the soul and essence of wisdom, the nature of the pursuit, the core elements of wisdom library, think beyond the technical level, to pay more attention to the creativity and information technology support for the development of the concept of "intelligence services".

Wisdom library is the continuation and development of the digital library, is through the Internet to provide users with a digital library intelligence service and management of advanced development, more wisdom. Wisdom library consists of people, resources, space of three elements, based on technology, to serve for the soul. Among them, one is the core of the three elements, resources and space to serve the people; technology is the foundation of wisdom library can be realized; elements and basic services through the wisdom of the library, closely associated with the people of this core elements.

People in the ideal model of wisdom library,, to the user demand, will be calculated into 
people's living space and information, between people and people, between people and resources, the human and the library (including physical and virtual space) between the library, resources, mutual communication between the Internet, at any time, any place, people can use a variety of ways arbitrary use of library, and obtain the intelligence service. Wisdom library in information technology supporting infrastructure, people, resources, space 3 elements can be fully aware and interconnection, in pursuit of green and sustainable development at the same time, intelligence services so that people can easily capture the required information and insight that information.

Characteristics of wisdom library

With the knowledge of wisdom library deeply, the author of wisdom library have a more thorough understanding. Wisdom library is mainly applied in intelligent sensing the environment and comprehensive information service platform, one is to provide personalized service based on role; each application and service areas of computer network based information services into the library, realize the interconnection and cooperation; two is through intelligent perception of the environment and comprehensive information service platform, to provide a mutual the exchange and mutual awareness interface for the library and the outside world. Wisdom library (Smart Campus) should have the following characteristics:

(1)The environment overall perception

Overall perception of wisdom library includes two aspects, one is the sensor can whenever and wherever possible perception, capture and transfer information about people, equipment, resources; two is the individual characteristics of learners (learning preferences, cognitive characteristics, attention, learning style and learning scenarios) (learning time, learning space, learning partner learning activity, etc.) acquisition and transfer of perception.

(2)Seamless interworking network

Based on the technology of network and communication, especially the mobile Internet technology, connecting intelligent library supports all the software and hardware equipment, information awareness can be rapid, real-time transmission, which is the basis for all users according to the new way of collaborative learning, collaborative work.

(3)Mssive data support

On the basis of data mining and modeling technology, constructing model based on "massive" data can be intelligent library, establish forecast method, trend analysis, and forecast on the new information; at the same time, wisdom library can be integrated in all aspects of the data, information, content such as regulation, through the intelligent reasoning, rapid response, take the initiative to deal with the characteristics, intelligent, wise more reflected.

(4) Open learning environment

The core concept of learning is the cultivation of innovation ability, the library faces from "closed" to "open" appeal. Wisdom library supports the expansion of resource environment, allow students to break through the traditional constraints; supports the expansion ring time, supports the expansion of space environment, let the library effectively use can occur in the real situation and fictitious situation.

Therefore, the wisdom of the library is a reader oriented personalized services for the concept, fully aware of physical environment, identify readers' individual characteristics and learning situation, and provide network communication seamless networking, effectively supporting the teaching process analysis, the open education teaching environment evaluation and intelligent decision making and convenient and comfortable living environment.

\section{Construction and application of three, wisdom library}

At present, our country is experiencing a profound social change, social life, pluralism and diversity. All libraries should have strong information consciousness, enthusiasm, to be familiar with the reader wondering what. Ensure that the library as a representative of advanced culture to fully reflect the requirements of the times. Only in this way, the library can always maintain the vitality and creativity, to the healthy survival and development. The construction of wisdom library which is based on the traditional library and digital library based entities, the network as the carrier, to realize intelligent management and intelligence service technology calculation using cloud library. 
Should not be completely abandoned the traditional entity library and many researchers have described, paperless library to realize virtual. The development of network technology should be based on the traditional entity library as the carrier, to achieve complementarities in the link of information storage, transfer, service etc.. Wisdom library to make the reader through the smart book borrowing system quickly finding the required books, but also get the book review and other digital information in intelligent terminals such as mobile phone, as well as published personal comments, the digital library service to a higher level.

Wisdom library network architecture consists of three layers: the perception layer, network layer and application layer.

(1) Perception layer

In perception layer mainly through the sensor, a two-dimension code tag, RFID sensing terminal, perception and acquisition information to the entity. For example, wisdom library through the sensor for temperature, the indoor light, smoke concentration, humidity sensing. The museum can automatically adjust the indoor climate changes according to the temperature to remain at the optimum temperature. Light induced brightness can be automatically adjust the indoor, in the rainy weather has a special regulation of the indoor brightness for all learning and reduce the damage to the eyes. When the indoor smoke reaches a certain concentration, sensor information, start alarm. The sensor and the monitor are connected, controlled by the monitoring system. The perceptual layer to complete the automatic perception on the books, the reader, shelves, other equipment and buildings.

(2)The network layer

The network layer is mainly composed of the Internet, network management system and the cloud computing platform, is the central nervous system of wisdom library, responsible for sensing layer information is processed and delivered to the application layer. The key to the network layer is the network protocol, such as a specific RFID reader wisdom library shelves, put in the books on the shelf tag. Reader and tag can be communicated by communication protocol specific information. The smart shelves can read out the details for each book, when the borrower borrow books, Book Smart frame can will borrow record go books and reflect to the books management system management system, a Book of less than a set value, the shelf will remind management system fill in time, at the same time the bookcase would do analysis of borrow books overall, the analysis results reflect to the management system.

(3) The application layer

The application layer is the practical application of wisdom library. Intelligence library borrowing system greatly improves the efficiency of the borrowed books. The lending library card can choose only, the system can automatically make the information and borrow books information record. The system will automatically check the return of books, if the book well into the book is normal, if the book is damaged the system will show the extent of the damage, and lists the compensation scheme. When some book has surpassed also the book deadline, the system can make the reminder automatically. Intelligent Book positioning system of wisdom library can help the borrower in the input to query the book, a three-dimensional map navigation accuracy for the loan, the borrower can quickly understand the book's storage and shelf. At the same time, through the entity library and digital library docking system, borrowers can use intelligent terminal further detailed information about the books, such as books and electronic books to read the comments. Intelligent Book inspection system can automatically complete the book to find, inventory, Shun frame, guide and other functions, and can provide information book lending rate to managements manage in order to add books, improve the efficiency of librarians.

\section{Conclusion}

Wisdom library implements the visualization management of library, characteristic of scientific, humane and intelligent. At the same time, wisdom library to build an intellectual environment, let the library extends to the real scene, the library and society, and the nature, and the readers visually connected together, between theory and practice in architecture for bridge, cultivating readers' scientific literacy and research ability, to stimulate their interest in exploring and sharing, 
strengthening the inner motivation human interaction and resources is of great significance in promoting the. The establishment and development of wisdom library is to optimize the knowledge environment, is the future direction of development. It has broad application prospects, and will face more challenges, including the concept, method of application, security and privacy, data protection, resource control, information sharing, the development of standards. Therefore, the development of wisdom library is not accomplish at one stroke, function and significance to correctly understand the wisdom of the library, and actively carry out exploration activities. The basic trend we must clearly understand technologies to promote the improvement of library, the construction and development of wisdom library to top design, do not blindly follow the trend, to suit one's measures to local conditions, because the museum system, to improve knowledge quality wisdom to create the environment as the fundamental purpose of library; library should update ideas, learn new techniques, in the Internet of things under the environment of new technology actively; technical service personnel should provide readers convenient, effective use of wisdom library to provide high quality service and efficient technical support; the reader should be happy to learn, active exploration, active learning techniques and methods in Library Science of wisdom, so as to cultivate their innovation ability and learning ability. The intelligent library has a broad application space and potential research value.

\section{References}

[1] Aittola M, Ryhanen T, Ojala T. Smart Library: Location-Aware Mobile Library Service [J].International Symposiumon Human ComputerInteraction with Mobile Devices and Services, 2003(5):411-415.

[2] Yan Dong. Wisdom library of Internet of things based on [J]. Library Journal, 2010 (7): 8-10

[3] The construction of Library and the service mode. The wisdom of [J]. intelligence information work, 2012 (5): 102-104

[4] Zhao Xiaofang. The way of wisdom library service implementation and construction of [J]. library and information, 2012 (6): 46-48 